\title{
Improving student accessibility, equity, course performance, and lab skills: How introduction of ClassTranscribe is changing engineering education at the University of Illinois
}

\section{Prof. Lawrence Angrave, University of Illinois at Urbana-Champaign}

Lawrence Angrave is an award winning Fellow and Teaching Professor at the department of computer science at the University of Illinois at Urbana-Champaign (UIUC). His interests include (but are not limited to) joyful teaching, empirically-sound educational research, campus and online courses, computer science, unlocking the potential of underrepresented minorities, improving accessibility and creating novel methods that encourage new learning opportunities and foster vibrant learning communities.

\section{Prof. Karin Jensen, University of Illinois at Urbana - Champaign}

Karin Jensen, Ph.D. is a Teaching Assistant Professor in bioengineering at the University of Illinois at Urbana-Champaign. Her research interests include student mental health and wellness, engineering student career pathways, and engagement of engineering faculty in engineering education research. She was awarded a CAREER award from the National Science Foundation for her research on undergraduate mental health in engineering programs. Before joining UIUC she completed a post-doctoral fellowship at Sanofi Oncology in Cambridge, MA. She earned a bachelor's degree in biological engineering from Cornell University and a Ph.D. in biomedical engineering from the University of Virginia.

\section{Mr. Zhilin Zhang, University of Illinois at Urbana - Champaign}

Zhilin Zhang is a 5-year BS-MS Computer Science student at the University of Illinois at Urbana Champaign (UIUC). He has experience in text retrieval and mining, machine learning, data analysis, mobile development, web development, and user study. His interests include (but are not limited to) HumanComputer Interaction, Artificial Intelligence, Data Retrieval and Mining, and Computer Security.

\section{Mr. Chirantan Mahipal, University of Illinois at Urbana - Champaign}

I'm a Computer Science grad student at University of Illinois, Urbana-Champaign, working under the mentorship of Prof. Lawrence Angrave. Prior to this, I was working as a Research Fellow at Microsoft Research in the Technology for Emerging Markets (TEM) group.

David Mussulman, University of Illinois at Urbana - Champaign

Dave is an Instructional Technology Facilitator with the University of Illinois at Urbana-Champaign's Engineering IT Shared Services. He helps instructors select and integrate technologies into their courses to enhance student learning and improve course administration.

\section{Prof. Christopher D. Schmitz, University of Illinois at Urbana - Champaign}

Christopher D. Schmitz is an Electrical Engineer and Teaching Associate Professor at the University of Illinois Urbana-Champaign. His research interests include algorithmic fault-tolerant adaptive systems, software defined radio, multi-user cellular communication, electrically-small devices, and pedagogies of teaching and learning. An amateur beekeeper, he currently mentors a project for improving the plight of honeybees.

He worked for TRW Space and Electronics Group from 1995 until 1997 and at the University of Illinois from 2002 to present. His research interests are in adaptive digital signal processing, digital communications, and education pedagogy. He currently serves the ECE department of the University of Illinois as a Teaching Associate Professor and an undergraduate advisor and is working to improve undergraduate education as an Education Innovation Fellow (EIF) in the Grainger College of Engineering.

Dr. Robert Thomas Baird, University of Illinois Center for Innovation in Teaching and Learning 
As Associate Director at the Center for Innovation in Teaching \& Learning Robert helps guide the testing, evaluation, and use of campus emerging technologies in the classroom and online. He has extensive experience in technology-enhanced classrooms, online teaching environments, and web-based student writing and multimedia projects. Robert teaches cinema, new media, and digital video courses. His 1995 dissertation used cognitive psychology to understand how filmgoers can be frightened and startled by film scenes they know are fictional. In the early 1980s he worked as an assistant film editor in Hollywood on over a dozen films. He has published essays on literature, film, and cognition. For the past 20 years Robert has been an active soccer player and coach, coaching youth teams for the local Illinois Futbol Club (Little Illini).

\section{Prof. Hongye Liu, UIUC \\ Mr. Ruihua Sui, University of Illinois at Urbana-Champaign}

Ruihua is a junior student in Mathematics and Computer Science at University of Illinois at UrbanaChampaign. He is the research assistant and frontend developer of ClassTranscribe. He was nominated for Illinois Innovation Prize 2020 because of his contribution to educational software.

\section{Dr. Maryalice S. Wu}

Maryalice is the Director of Data Analytics at the Center for Innovation in Teaching \& Learning at the University of Illinois Urbana-Champaign. She holds a position as an adjunct assistant professor in the Department of Sociology at the University of Illinois and has a Ph.D. in Sociology from the University of Illinois. Her recent research focuses on the economic and health empowerment of women in developing nations. Her other projects relate to program evaluations and learning analytics in academia. . She has taught several courses at University of Illinois, including Introduction to Social Statistics, and Social Research Methods.

\section{Mr. Rob Kooper, NCSA / University of Illinois at Urbana Champaign}

Rob Kooper is a lead research programmer at the software directorate at the National Center for Super computing Applications. He is interested in enabling scientists to do research work using software developed with the help of NCSA as well as teaching good software principles during this process. He is interested in software deployment and scaling software deployments from small research projects to larger installations with many users. 
ASEE 2020 - New Engineering Educators Division (Full Paper)

\section{Improving student accessibility, equity, course performance, and lab skills: How introduction of ClassTranscribe is changing engineering education at the University of Illinois}

\section{Abstract}

This paper presents three case studies that examined the use of ClassTranscribe in a diverse set of undergraduate engineering classes in 2019 and 2020 at the University of Illinois at Urbana-Champaign. ClassTranscribe, a video viewing system designed with accessibility and learning in mind, was first presented to the ASEE community in 2019 [1]. The pedagogical features of the tool include: pause, leave and resume viewing; captions available in multiple languages; downloadable transcriptions; shareable links to video moments; and searchable content within each video and across an entire course. The importance of the tool in the context of a commitment to accessibility for engineering education is discussed. In addition, online video content that follows Universal Design for Learning principles enables all students to prepare for assessments and provides more equitable learning opportunities. Low-stress online learning opportunities may be particularly valuable for underrepresented minorities (e.g., [2] explored relative student improvement in multiple exams assessments in an online course with video lectures). The first study presented here describes two extremes: a supplemental-resource approach and primary-resource approach in the adoption of ClassTranscribe into an Electrical and Computer Engineering (ECE) and Computer Science (CS) course, respectively. In Spring 2020, ECE students used the tool as a supplemental learning resource to review and search live lecture captures. Whereas in the sophomore CS course ( $\mathrm{N}=271$ students), physical lectures were canceled and ClassTranscribe was used as a primary learning resource using short-length $(<10$ minute) videos. The methodology and analysis of the CS course outcomes were described in [3] but relevant findings are reproduced here: Students in the lowest quartile improved by 3.3 percentile points compared to previous semesters and statistically-significant positive learning outcomes were found at all levels of student performance. Each quartile of students who used the tool an above average amount of time led to increased final exam scores, with the lowest quartile increasing 14.4 absolute points compared to their in-quartile peers. A new contribution of this paper is an analysis of the interactions with the tool, which found three event types:

Fullscreen-viewing, Change-playback-speed and Search-Transcription predicted improved exam performance and were statistically significant $(\mathrm{p}<0.01)$. In the second case study, video instruction was used to present safety and lab-handling techniques in a Bioengineering class prior to rigorous lab assessment. The student-specific analytic data available to the course instructor using the web interface allowed the instructor to check that each student had 
completed training materials prior to lab work. Student surveys measured several factors including the utility of the tool and students' preferences in video systems and video-based Bioengineering instruction. In the third case study, student feedback from CS and ECE courses were solicited on learning utility and digital accessibility. The majority of the 206 responses reported high utility ("moderately useful" or greater) of the tool when working on assignments $(67 \%)$ and preparing for an exam (77\%). The majority of respondents $(96 \%, n=239)$ recommended the tool be re-used in future semesters of their course. We present example survey questions that respect student choice to not-self disclose a disability.

\section{Introduction}

This paper presents the educational impact of adding accessible video content using an accessible video player to courses in 3 undergraduate engineering majors: Bioengineering, Computer Science and Electrical and Computer Engineering. The web interface of the open source video platform, ClassTranscribe, was designed and created specifically to enhance student learning; support students with disabilities including vision, auditory, motor and cognitive disabilities; and provide detailed behavioral per-student data that is relevant to students, instructors and educational researchers.

This paper is organized as follows. The background section provides a broader context of the work presented here: i) Motivation for the need to improve digital accessibility in engineering education, ii) The challenges of providing equitable access to all students, iii) Use of video for learning, iv) How application of Universal for Design for Learning principles and online content may provide greater equity and access, v) The limited understanding of imposter syndrome effects and interventions in engineering education and the potential to improve students' self-confidence. Three case studies are presented. The first case study described the use of the tool in CS and ECE courses and analyzed which student behaviors lead to statistically-significant favorable learning outcomes. The second case study examined the use of ClassTranscribe to address challenges of a Bioengineering laboratory course and student preferences for further use of ClassTranscribe-based learning in Bioengineering. The third case study surveyed students to gain insights into accessibility and utility of the accessible video from the perspective of engineering students who used the tool in computing and computing engineering contexts.

\section{Background}

The National Center for Science and Engineering Statistics (NCSES) publishes detailed tabular data on the demographic characteristics, enrollment, degrees, and employment of women, minorities, and persons with disabilities in science and engineering (see Table 2-6 of [4]). In 2016, using the NCSES definition, "any type of disability related to blindness, deafness, severe 
vision or hearing impairment, substantial limitation of mobility, or any other physical, mental, or emotional condition that lasted 6 months or more," there were 3,807,100 students classified as disabled and enrolled in college (52\% of those are in 4-year programs), which represents approximately $19 \%$ of the total undergraduate enrollment in the U.S. Of which there were 203,793 disabled students enrolled in engineering, excluding computer science (Table 2-7 of [4]). For 2015, engineering, computer science or math undergraduate degrees, the NCSES reports $19.6 \%$ of students have a disability (Table 311.10 of [5]). So, where are they?

Though some disabilities are visible, many are invisible. An instructor may be unaware that they have students with color-blindness, low vision, partial deafness, low mobility, low dexterity, attention deficit hyperactivity disorder (ADHD), attention deficit disorder (ADD), autism, tinnitus, dyslexia ..., and other physical and cognitive impairments not listed here that a student does not need to disclose. Further, a 2017 study at a Wisconsin university found that $75 \%$ of students chose not disclose their disability to their university [6] and preferred their impairment to remain invisible. Respecting non-disclosure in student surveys is also discussed in the third study below.

The design of today's digital interfaces and the digital content rarely consider impairments and disabilities. In short, they are digitally inaccessible. Practical, comprehensive digital accessibility remains an elusive problem across multiple engineering education fields. Over time, each technological barrier further reduces the talent pool and ultimate diversity and ingenuity of our future commercial workforce and academic faculty. For example, modern CS-STEM ${ }^{1}$ positions report less than $1-2 \%$ of their workforce identify as disabled, and some high profile companies prefer to remain silent [7]. The education pipeline for engineering students is ripe for improvement.

The utility of online videos, online courses and entire online programs offered at scale (Coursera, edX) has been, and continues to be, the subject of innovation, scholastic research and criticism (e.g. [3], [8]-[10]). In this paper, we consider the availability of accessible video content as an application of the first principle of Universal for Design for Learning (UDL) (e.g. [11] [12], [13]). UDL's three principles are: “1) multiple methods of representation that give learners a variety of ways to acquire information and build knowledge; 2) multiple means of student action and expression that provide learners alternatives for demonstrating what they have learned; and 3) multiple modes of student engagement that tap into learners' interests, challenge them appropriately, and motivate them to learn." Some advantages of UDL to students in engineering courses and the benefits to the university are presented in the Conclusions section below.

\footnotetext{
${ }^{1}$ Computer science, science technology engineering and math disciplines
} 
Engineering courses with rigorous assessments of demanding technical skills are an intimidating barrier to unconfident undergraduates. A lack of confidence and limited belief in long-term success in a major, can undermine a student's success in a chosen major. These negative factors are more common in underrepresented minority students and thus, may ultimately reduce retention of underrepresented minorities in an engineering major, and/or nurture imposter syndrome. Imposter syndrome is a persistent cluster of negative self-beliefs (first studied in interviews with high achieving women [14]) that affects men and women equally[15], where the person does not identify as a valid member of a group, and attributes their successes to short-term luck or hard work, rather than their competence and ability. Imposter syndrome has been widely studied in academic and professional fields and is positively correlated with mental health concerns of underrepresented students [16] and [17]; see [18] for more examples. In the context of engineering education, [19] describes an instructor's personal experience and suggestions of interacting with chemical engineering students with imposter syndrome but scholastic research on successful imposter syndrome inventions for engineering is scarce. Addressing imposter syndrome and providing multiple pathways to success may help with retention of all students but may especially help with equitable access for underrepresented students. This includes engineering disciplines with underrepresented gender and LGBTQ* gender identities [20], and ethnic and racial minorities, first generation students and students with disabilities. Availability of online videos is one mechanism that promotes students' agency in their own educational activities, and allows multiple low-stress opportunities to learn and review material prior to high-stress, high-stake assessments. Future scholastic research into causal relationships and the efficacy of interventions to reduce imposter syndrome in undergraduate engineering education is worthy of future study. For the scope of this paper, the authors defined a more conservative hypothesis: Opportunities for low stress learning experiences prior to challenging assessments that lead to successful assessment outcomes will encourage students to be positive about their choice of major, and more self-confident in their future ability to succeed in their major. Though we do not examine learning outcomes by demographics, we note that equitable access to course video content can significantly lower the stress surrounding a course because students can watch and review content they did not at first understand and without judgement or comparison to their peers.

Study I. CS \& ECE classes replaced and supplemented in-lecture activities

In 2019 and Spring 2020, several computer science (CS) and electrical and computer engineering (ECE) faculty used Class Transcribe in their courses. For this study we analyze and report in detail on a large enrollment introductory ECE class and sophomore-level CS system programming class. 
The ECE "Introduction to Electronics" course goals are to excite students about the study of electrical and computer engineering by exposing them early in their education to electrical components and their application in systems, and to enhance their problem-solving skills through analysis and design. ClassTranscribe was first made available in the course during Fall 2019. There were 52 students who chose to use ClassTranscribe to also watch the recordings of the live lectures, approximately $13 \%$ of the total enrollment. In Spring 2020, students were again provided recorded lectures using ClassTranscribe. At mid-semester, the attendance requirement was dropped and students were allowed to attend a live, synchronous lecture or watch the ClassTranscribe videos asynchronously. Students could use ClassTranscribe anonymously, sign in using a personal gmail, or sign in using their university email address. The number of video minutes was only tracked in the latter case. Video minutes viewed were logged in 15 second increments. The histogram of student use of ClassTranscribe, when they used their University email, is shown in Figure 1.

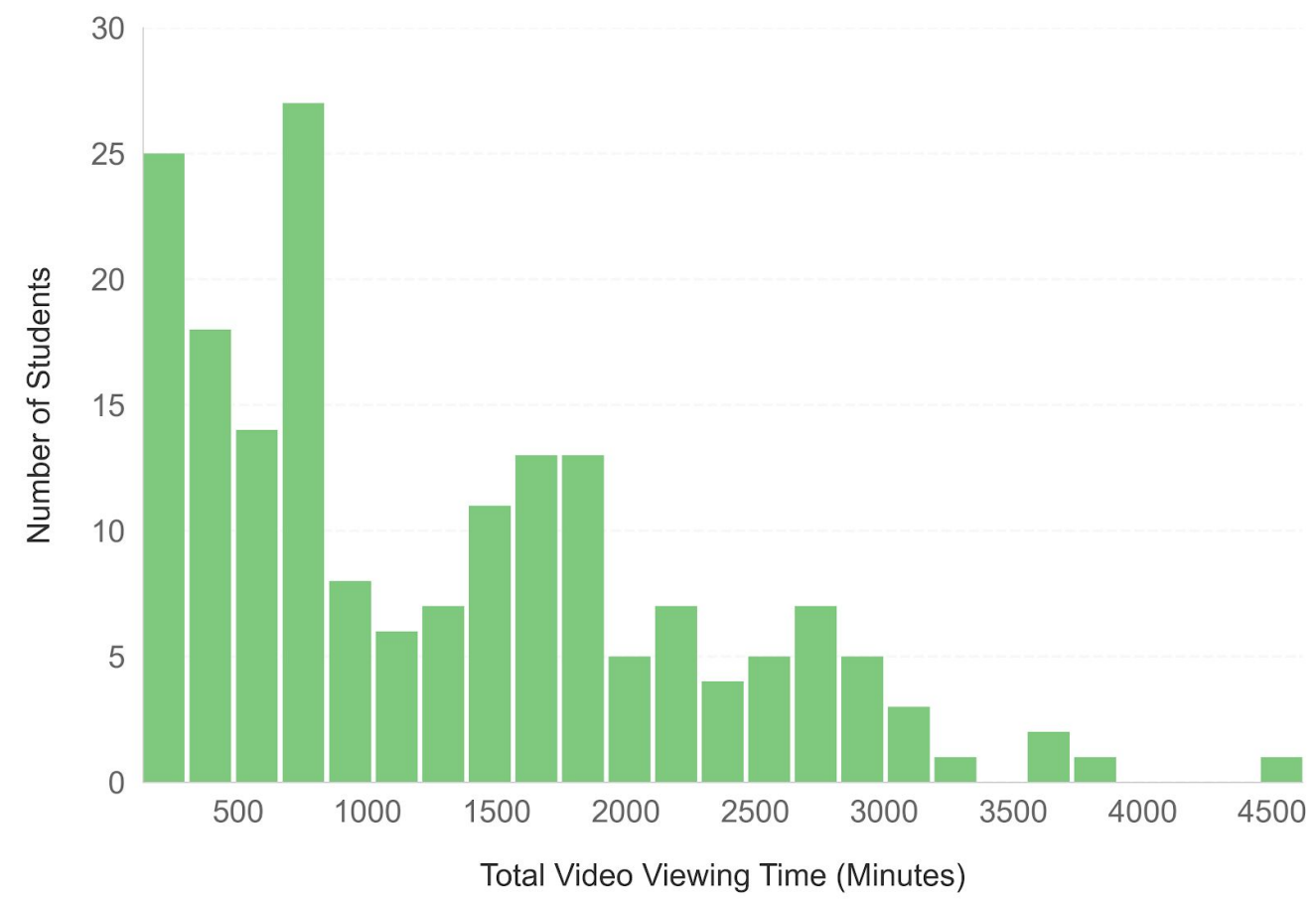

Figure 1. The histogram of total video-minutes viewed by enrolled ECE students when ClassTranscribe was offered as a supplemental component. One student watched 4627 minutes of video, approximately double the actual content length. The total number of ECE students may be slightly higher than can be inferred from this figure because we report only students who signed in using their university email and excluded users who were anonymous or used a personal gmail address to sign in. 
Considering students who used the system at least once, a linear regression found a statistically significant effect $(\mathrm{p}<0.04, \mathrm{n}=231)$ where minutes-viewed predicted the student's course total score: Students who watched at least 2000 minutes (equivalent to 40 lectures, each 50 minutes) were predicted to increase their course total by 2.4 absolute points on a 100 -point scale, i.e. approximately one letter grade improvement (e.g. B to $\mathrm{B}+$ ). This linear regression is illustrated in fig. 2 (upper line).

ClassTranscribe was used in a supplemental manner, and many students were capable of achieving high scores without it (for example, review the prevalence of high scores near the upper-left area of fig. 2). To further analyze these data, we assumed an additive learning model (i.e., students could elect to perform additional, uncorrelated actions that further increased their competency and skills), which could be performed independently of measured ClassTranscribe use. Thus, for a known amount of Class Transcribe use, the minimum observed score would represent the expected student ability with other independent additive processes minimized. One could measure the observed minimum score for evenly spacely binned ranges of video minutes $(0,500,1000 \ldots 5000)$. However even-width bins would introduce an artificial sample bias because the observed minimum of a particular bin will be statistically biased to a greater value if the bin contains fewer samples than other bins. Thus, the data was split into ranges of equal sample counts $(n=25)$. The bin ranges are indicated by the gray vertical lines in Fig 2 . Using a linear regression, ClassTranscribe-use was found to be statistically significant predictive of improved course scores $(\mathrm{p}<0.02)$ when considered as an independent learning activity under an additive learning assumption. For example, a student that viewed the complete course (2000 minutes) using ClassTranscribe is predicted to achieve at least $73 \%$, a passing score, independently of other uncorrelated factors that may further improve their course score. We next discuss Class Transcribe in a Computer Science course. 


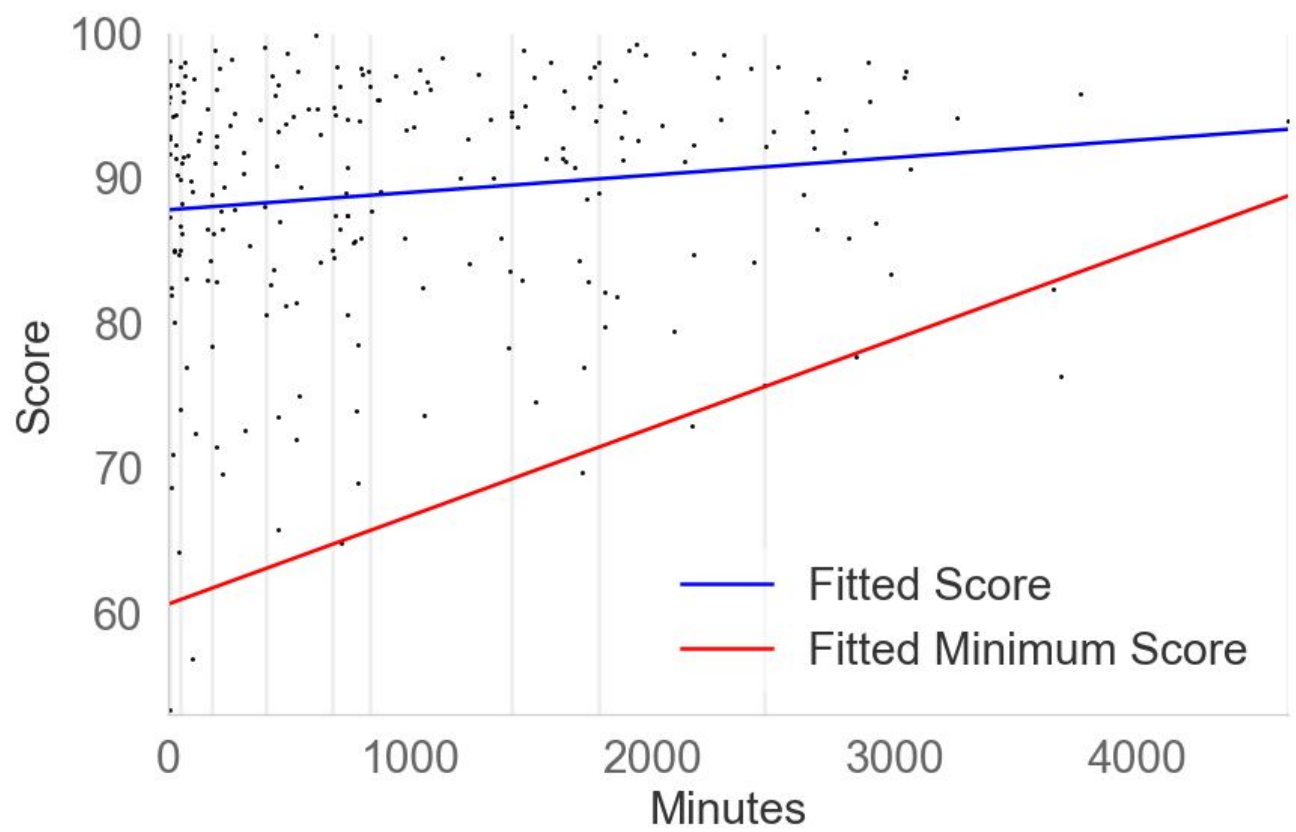

Figure 2. A scatterplot of the student course totals for each ECE student that used ClassTranscribe at least once using their university email. Two statistically significant effects were found: The average course score improved by 2.4 points and minimum expected course score increased from $61 \%$ to $72 \%$ when students watched the equivalent of the full course videos (2000 minutes).

The significantly positive effects on learning outcomes of adopting ClassTranscribe in a Spring 2019 semester large enrollment ( $=271)$ Computer Science sophomore-level core-curriculum system programming course has recently been reported in a 2020 paper [3] and are summarized here. The class cancelled regular physical lectures scheduled at 8 a.m. and instead used ClassTranscribe to provide web-based video lecture content. A subsequent analysis of students' course performances found that all quartiles of the students' course scores improved compared to students in all previous equivalent semesters. For example, the lowest quartile of the course scores improved by 3.3 points compared to the previous Spring semester $(83.0 \%$ vs $80.0 \%)$. With other course factors held constant over multiple past semesters (including unique final exams that were derived from a large random question set, course format, syllabus and instructor), the instructor concluded that at least the "First do no harm" principle had been satisfied by the adoption of the tool to deliver course content in a video format.

A concern for educators and educational researchers is that course interventions and new opportunities presented to students are only utilized by the already highly-engaged, highly-motivated ("A" and "B" grade) students while leaving the remaining struggling students unaffected, or only weakly affected at best. Fortunately the adoption of this tool had the opposite 
effect: Student behaviors were analyzed and the tool's impact on the students' learning outcome were inferred by recording detailed student interactions over the last 6 weeks of the course. A statistical analysis in the same paper examined the effect of video minutes-viewed on improving final exam performance, by using course performance (excluding final exam score) as an independent variable. The large class size provided an opportunity to statistically analyze each quartile group separately. For each quartile, the students that used the tool an above average amount compared to their peers in the same quartile, were compared to students that used the tool less. All students who used the tool an above average amount improved compared to their peers in the same group (and this result was statistically significant), but the improvement was largest for the lowest two quartiles. The lowest quartile group improved their average exam score by 14.4 absolute points on a 100 point scale. A Mann-Whitney $U$ test, which is similar to student $\mathrm{t}$-test but does not require an assumption of normal distribution [21], found all improvements were statistically significant $(\mathrm{p}<0.03)$.

For each group, the above-average users of the tool exhibited a fractional improvement by reduction of $30-40 \%$ of final exam missing points, compared to their peers in the same quartile. These results supported the finding that use of ClassTranscribe is an effective learning technique that is open to students of all competency levels. The authors suggested this could be communicated to students as "Want to do well in the final? Take time to watch the videos over the semester and you can reduce your exam points you would have lost by a third."

The above paper only analyzed the total video minutes viewed by each student and did not analyze other student behaviors recorded by the tool. In this paper we describe the 13 different event types and examine other student behaviors. Specifically, we ask if event types were statistically predictive of improved student outcomes in the course.

\begin{tabular}{|l|l|l|}
\hline Event Type & Count & Event Description \\
\hline timeupdate & 413912 & Watched another 15 seconds of video \\
\hline play & 164428 & Selected the "play" button to play a video \\
\hline seeking & 160924 & Adjusted the video playback position. \\
\hline seeked & 102531 & Changed to a different part of the video \\
\hline pause & 153034 & Selected the "pause" button to pause playback \\
\hline userinactive & 134455 & Switched focus away to another webpage \\
\hline changevideo & 24750 & Changed to a different video \\
\hline
\end{tabular}




\begin{tabular}{|l|l|l|}
\hline changedspeed & 23151 & Changed the playback speed \\
\hline fullscreenchange & 17195 & Selected full screen mode to watch a video \\
\hline selectcourse & 2850 & Selected a course \\
\hline filtertrans & 1087 & Updated search filter of the transcription interface \\
\hline edittrans & 83 & Edited a transcription \\
\hline sharelink & 13 & Generated a shareable URL of the current position \\
\hline
\end{tabular}

Table 1 . The 13 event types captured.

The authors expected that students would post hyperlinks into an external discussion forum to discuss the most confusing or interesting moment of lecture. However the sharelinks feature was used only 13 times. Peer and forum-based discussion is an important pedagogical aspect of engagement and learning; therefore, a future version of the tool will promote the existence of this feature when students are first beginning to use the tool. Later surveys (see study II and III) also supported the finding that students were unaware of existing features beneficial for learning.

We hypothesized that some events (e.g., using full screen playback) might be a proxy for a student committing their full attention to the content, and thus changing to fullscreen would be predictive of improved student outcomes. Other behaviors (e.g., pausing the video or switching to another activity) might be indicative of either partial attention and disengagement, or integrated behaviors where a student is using the video player in conjunction with another course-related activity (e.g., working on an assignment). No statistically-significant event measures (criteria Mann-Whitney $U$ test, $\mathrm{p}<0.05$ ) were found that impaired exam performance. In addition to measuring the total video minutes viewed, searching for content, changing playback speed and using full screen were behaviors rejected by the null hypothesis (Mann-Whitney $U$ test, $\mathrm{p}<0.01$ ) and predicted further improvement of final exam performance (see Table 2 and Fig. 3). Other event types had no predictive power (i.e., could not exclude the null hypothesis). For example, there was no evidence that pausing the video led to either improvement or impairment of final exam performance. Acknowledgement: These results were based on original work by Zhilin Zhang as part of his 2019 undergraduate thesis.

\begin{tabular}{lllllll}
\hline Behavior & $\mathrm{N}(\mathrm{Y} 0)$ & $\mathrm{N}(\mathrm{Y} 1)$ & mean $(\mathrm{Y} 0)$ & $\operatorname{mean}(\mathrm{Y} 1)$ & threshold & p-value \\
\hline Search Captions & 185 & 86 & $79.5 \pm 13.4$ & $83.4 \pm 12.5$ & 0.0 & $<0.01$ \\
Change Speed & 199 & 72 & $78.4 \pm 13.7$ & $87.2 \pm 9.4$ & 113.2 & $<0.01$ \\
Full Screen & 212 & 59 & $78.9 \pm 13.7$ & $87.5 \pm 8.8$ & 81.5 & $<0.01$ \\
\hline
\end{tabular}


Table 2. Behaviors that were predictive of exam performance. The mean and standard deviation for the final exam scores for students that included above threshold (group "Y1") or below or equal threshold (group "Y0") of the described behavior. Searching captions, changing playback speed and using full screen options were correlated with improved final exam score (approximately 4 - 8 points improvement).

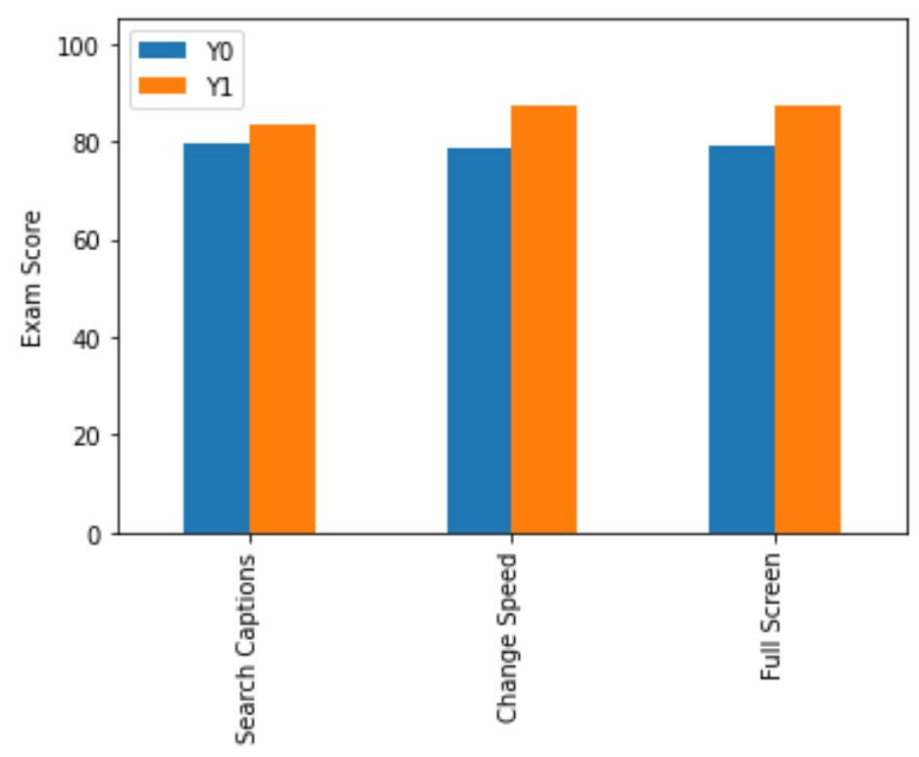

Figure 3. Student behaviors that were predictive of improved learning. Students who searched the transcriptions, changed video playback or used the full screen features more than average (Group Y1) than other students (Group Y0), had higher final exam scores than those who did not. See Table 2 for details.

Study II. Improving student outcomes in a Bioengineering lab safety and skills course

In Spring 2020, ClassTranscribe was used in a required sophomore course for Bioengineering majors. The course included principles of cell biology inherent in tissue engineering design and lab experience in safely and skillfully manipulating mammalian and bacterial cells.

The course included a lab practical exam that tested student ability to culture mammalian cells. As part of the practical exam, students are also required to use a brightfield microscope. Students are assessed on their ability to work in a biological safety cabinet (BSC) aseptically, and their ability to trypsinize cells, count them on a hemocytometer using a brightfield microscope, and successfully plate a new culture. Lab practical grades are assigned based on properly following a protocol, technique, following safety procedures, and use of the lab equipment (BSC and brightfield microscope). To prepare students for the lab practical exam, the course instructors 
created and distributed two training videos for students to use in preparing for the practical exam. The first video showed a student completing the protocol, with voiced explanations of each step. The second video demonstrated use of the brightfield microscope, including all dials to make appropriate adjustments (see Fig. 4).
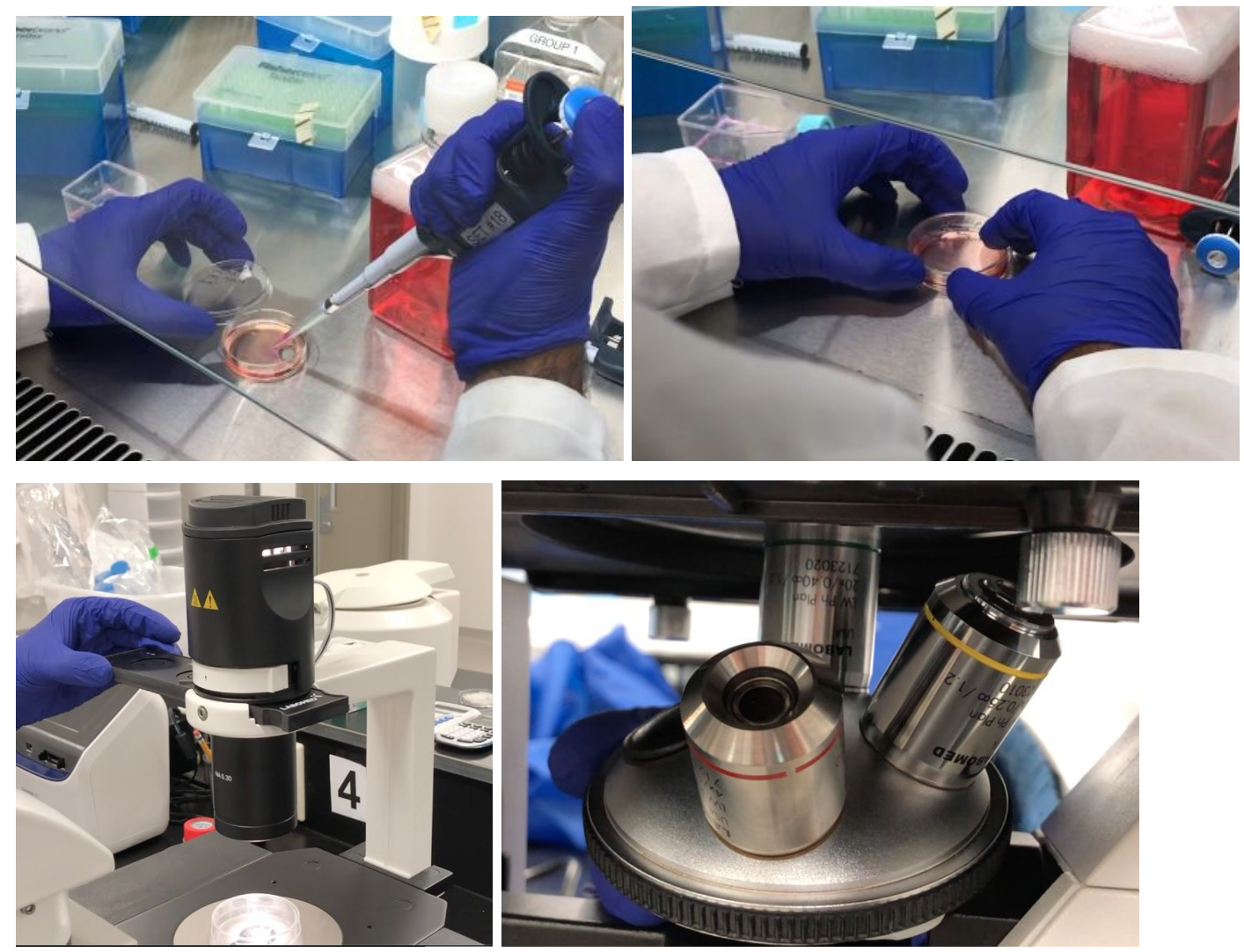

Figure 4. Screenshot examples of the training videos.

Video attribution: Videos created by Benjamin David and Faisal Masood.

The instructor analytics interface was used to verify that each student had watched the required videos.

Students were also surveyed about the inclusion of online videos that were available outside of the lab time to watch and learn the practical skills and to understand the safety aspects of the lab. The survey included free response items and 5-point Likert Scale for response scales. There were 11 respondents, though 2 were discarded because the respondents were unsure about which video system they used and their answers to subsequent questions were considered unreliable. The small number of responders is a limitation on the strength of the results presented below.

Of the 9 responders, 7 reported the text search feature as "Very Useful" (highest scale response), though only half $(n=5)$ were aware that the feature existed when using the tool during the course. 
ClassTranscribe was the most preferred system ( $\mathrm{n}=3)$, followed by YouTube/Box $(\mathrm{n}=2)$. No student preferred the University-licensed commercial systems (Kaltura and Echo360).

All students responded positively to "Should Class Transcribe be used in BIOE 202 in the future? [Yes/No]" Students also suggested other BIOE classes that could benefit from ClassTranscribe, with "BIOE 206" (a sophomore Cellular Engineering lab course) being a popular suggestion.

Free-form comments included feedback about the user interface and the utility of the search interface and being able to find the desired information. Some examples are below.

"I love having subtitles on the other side of the screen so I can see what information is being presented in the current slide. Seeing what has been presented or is going to be said helps a lot as well, as it helps provide context. I also like being able to search for certain term."

"The text feature is really nice"

"I really like the search feature within the transcript to find the info I need quickly."

"It was really helpful to be able to jump around by text keyword (even though not all the captions are very accurate) especially when looking for something specific from lecture to aid in completing an assignment. Much more efficient than jumping around aimlessly"

We asked students to reflect on how useful ClassTranscribe was for learning, preparing exams and working on assignments. Students reported favorable and similar utility in all three categories (see the Lickert results presented in Fig. 5). Only one respondent chose "Not at all useful."

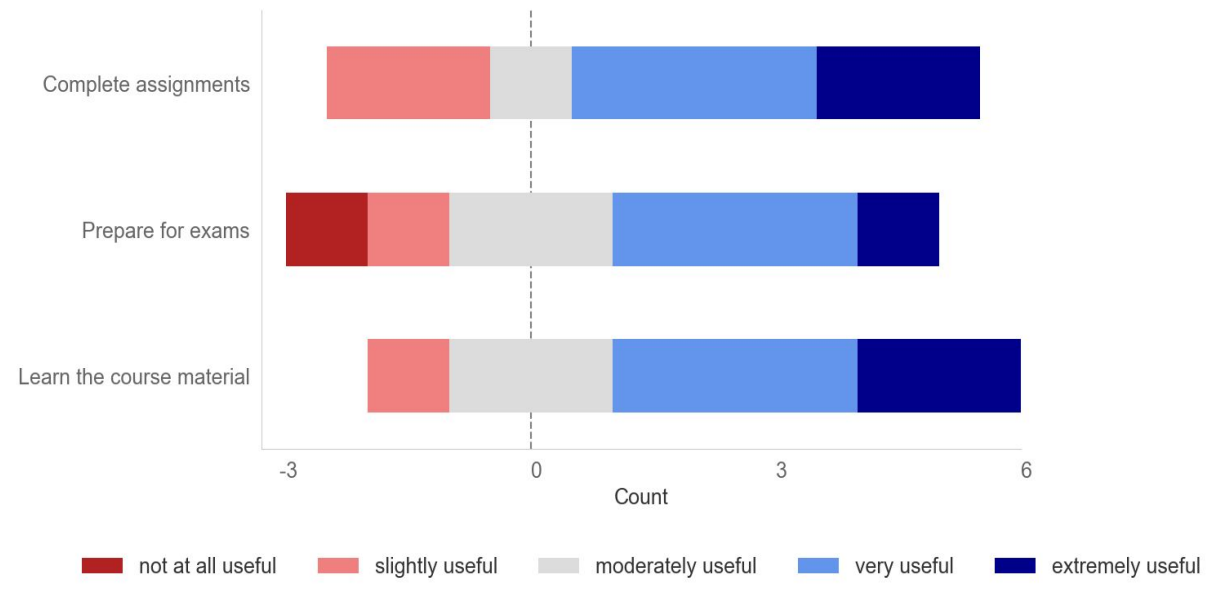


Figure 5. Survey responses to the utility of ClassTranscribe for learning, preparing for exams and working on assignments in a bioengineering sophomore required laboratory course. Note for comparative visualization purposes, we conservatively represent "moderately useful" as a neutral response.

These results are congruent with the survey results from earlier surveys in ECE and CS courses that have larger sample sizes which we report in the next section.

The instructor also noted that behavioral analytic data in the form of downloadable event data and data visualizations enabled the instructor to identify when students stopped engaging with the course, discover the most viewed content and enumerate frequent and infrequent search queries.

Study III. Student feedback on learning utility and digital accessibility

To assess the perceived learning utility and digital accessibility for electrical and computing-related courses, students in a Fall 2019 ECE introductory course and CS system programming course in Study I were surveyed. In the Fall semester both courses had in-class lectures and written materials available (i.e, use of the tool as a primary or secondary resource was entirely optional). Surveyed students reported on the utility of the tool using a 5-point scale for different learning activities which we summarize below in Table 3. The top-3 survey responses ("Extremely / Very / Moderately useful") were included in the numerator of the percentages below. A greater number of CS students responded to the post-semester survey because those students were offered a nominal 1 extra bonus point in the final exam. A third-party university survey service ensured student anonymity.

\begin{tabular}{|l|l|l|l|}
\hline $\begin{array}{l}\text { How useful was the video } \\
\text { content for - }\end{array}$ & $\begin{array}{l}\text { ECE } \\
\text { Respondents }\end{array}$ & CS Respondents & Combined \\
\hline - Learning the course material? & $100 \%(\mathrm{n}=15)$ & $89 \%(\mathrm{n}=191)$ & $90 \%(\mathrm{n}=206)$ \\
\hline - Preparing for exams? & $100 \%(\mathrm{n}=15)$ & $76 \%(\mathrm{n}=191)$ & $77 \%(\mathrm{n}=206)$ \\
\hline - Help completing assignments? & $93 \%(\mathrm{n}=15)$ & $64 \%(\mathrm{n}=191)$ & $67 \%(\mathrm{n}=206)$ \\
\hline $\begin{array}{l}\text { Should ClassTranscribe be used } \\
\text { in this class again? }\end{array}$ & $100 \%(\mathrm{n}=24)$ & $96 \%(\mathrm{n}=215)$ & $96 \%(\mathrm{n}=239)$ \\
\hline
\end{tabular}


Table 3. Summary of students' opinions of the tool's utility for different activities and for the course as a whole.

Free form responses included "I have a hard time understanding content right away in class so being able to watch lectures with the ability to speed up, slow down, and pause, is super beneficial to my learning... it was great!" When asked what other classes would benefit suggestions included both lower and upper-curriculum ECE and CS classes and more general suggestions, "all classes its super useful" and, "All senior citizens should have Life Alert and all STEM classes should have ClassTranscribe."

The most surprising result to the authors was that only $40 \%(96 / 242)$ responded affirmatively to "Were you aware of ClassTranscribe's ability to search for course content using text search?" This supports the finding discussed earlier, that students are often unaware of learning aids in digital user interfaces. To address this deficit future versions of the tool will provide a short guided introduction to the functionality and the authors will continue to use surveys and behavior analytics to review student use of the learning features.

For educators interested in understanding accessibility and disability needs of their students, we note that care is required to respect students' desire to not disclose their own disability. Thus accessibility and disability related survey questions in this study were framed from a broader perspective than merely the student's direct personal experience or disability-

"Imagine that you or another classmate had a physical (e.g., hearing, vision, motor) or cognitive (e.g., ADD, ADHD, dyslexia) impairment. How useful do you think ClassTranscribe would be for a student with an impairment?"

"How can we improve ClassTranscribe to be a better accessible learning tool? What feature(s) would increase the learning value of ClassTranscribe to you or to people with physical or cognitive impairments?"

Results from the survey and feedback from disability experts were further used to improve the interface. The original and revised interfaces are shown below (Fig. 6 and Fig. 7). The original interface always displayed the captions under the video area. 

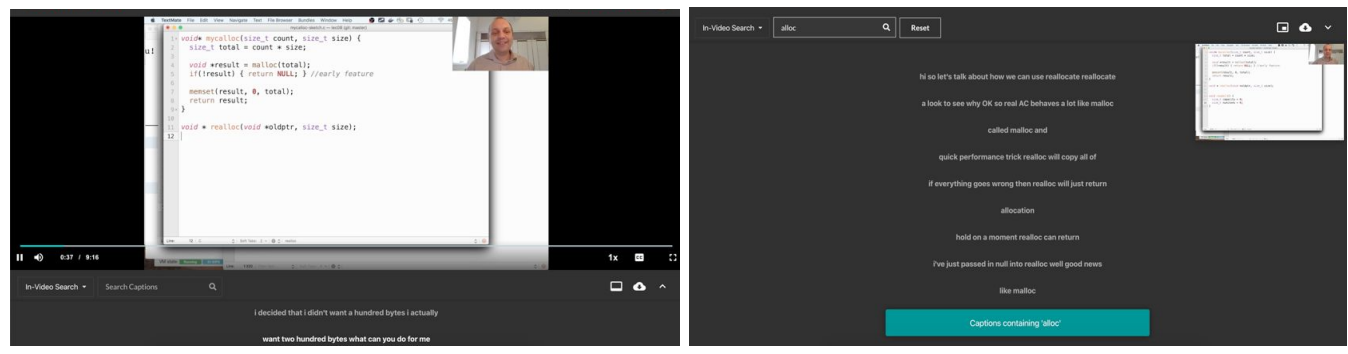

Figure 6. Two examples of the original 2019 web interface. The left screen capture shows a video playback with captions underneath. The right screen capture shows searching the video content using the captions. As the student types in a search term, matching caption lines from the entire set of course videos were immediately displayed.
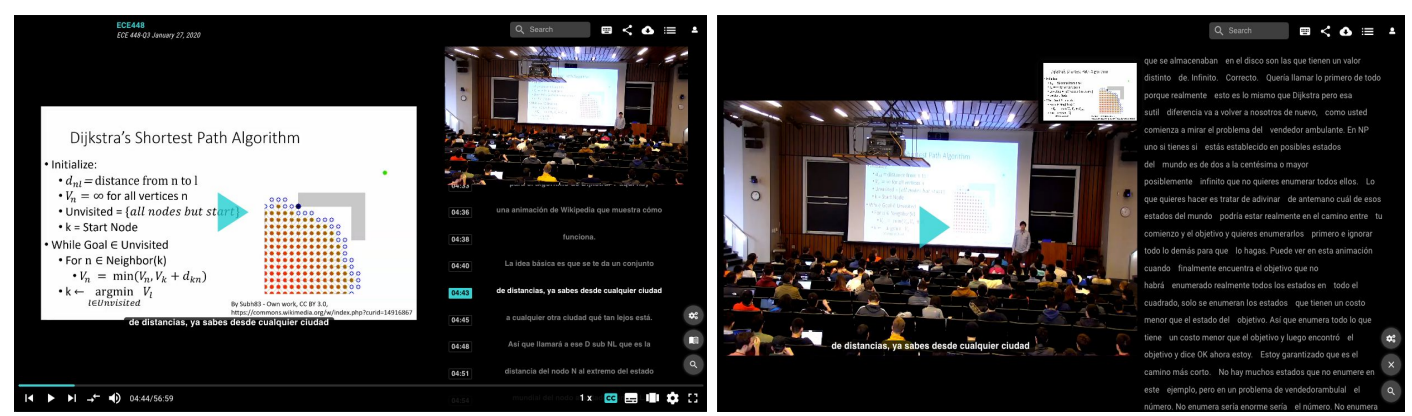

Figure 7. Two examples of the redesigned accessible 2020 video interface. The new video system provides far greater configurability of playback, which is particularly important for students with ADHD and ADD. For example, playlists, captions and transcriptions can be configured or hiddens, to help students concentrate on the content. In the screen capture above a non-native English student preferred to watch the videos with automated Spanish transcription (translations to Korean, Spanish and Simplified Chinese were provided). Configurable caption font size and color, background color, background transparency to assist deaf students and students with low vision, cognitive and reading difficulties (e.g., dyslexia). Text searching across the current video or the entire video set is now intuitive.

Future work

A student may exhibit several behaviors described in study I, and the importance and interaction of multiple behaviors recorded by the tool is worthy of further investigation in single courses and multiple types of courses. 
In this report we were not able to directly measure learning outcomes specifically for students with disabilities and International students. Future research will address these limitations.

Improving digital accessibility is a continuous process and the tool developers will continue to work with disability experts and seek feedback from students with impairments.

The next version of the tool currently in development can create accessible ePub (digital books) directly from video lectures. We look forward to reporting more on this work in a future paper.

\section{Conclusions and discussion}

In this paper we reported on three studies that focused on examples of adding ClassTranscribe into multiple engineering classes with multiple goals of improving digital accessibility, self-efficacy in the course (i.e., self confidence and self-beliefs in succeeding in their major) and effectiveness (at all levels of student ability). Learning outcomes and examples of adoption were presented under a diverse set of educational uses including use as a primary source of lecture content (CS, Spring 2019), secondary or supplemental review of recorded live lectures (ECE,CS, Fall 2019,Spring 2020) and pre-lab training for lab techniques and equipment use

(Bioengineering, Spring 2020). Per-student learning data was used with gradebook data and demonstrated statistically significant positive learning outcomes in multiple courses. Behavior analytics and survey responses identified that some students were unaware of learning features and future versions of the tool could introduce the interface to the user. In all courses surveyed, BIOE, CS, and ECE students overwhelmingly recommended the use of the tool in future semesters. The majority of CS \& ECE respondents found the tool moderately, very or extremely useful for both homework (67\%) and exams (77\%), with similar results in the smaller BIOE survey. Carefully worded surveys that respected a student's right to not self-disclose disabilities were used to identify opportunities to improve accessibility features, which are being addressed in the next version of the tool.

In closing, we summarize the benefits of why an educator or institution may consider adoption of ClassTranscribe and the use of searchable, accessible video content. From a Universal Design for Learning perspective [22],[23], low cost, accurate captions and transcriptions benefit not only students with hearing impairments, but also International students with imperfect English listening skills, blind students who listen to transcriptions using text-to-speech at high speed $(>3 x)$, and all students who want to search for relevant content, review content multiple times, or are in a noisy environment. Interfaces designed for ADD/ADHD may help all students focus and concentrate. Ability to choose font style, size and colors may help those with visual impairment or dyslexia. An interface that is fully accessible using keyboard alone benefits students with motor impairments and aids all students to navigate efficiently using keyboard shortcuts. 
Searchable online content is the de facto behavior of today's learners. Providing means for students to find relevant video content can only help students prepare for assessments, homework and exams. Language translations further aid non-native English speakers and allow high quality engineering education content to reach a greater audience, and help set a new level of quality for engineering outreach content. From a competitive standpoint, the authors hypothesize it may further encourage top international and domestic students and students with disabilities to apply to US institutions that visibly assist all students to thrive in their educational program.

Accessible access to online video content is one step towards equitable access for all students in engineering education. We invite engineering educators interested in adopting the tool to contact ClassTranscribe@classtranscribe.illinois.edu.

Acknowledgements

We also wish to acknowledge UIUC IT staff, NCSA, the College of Engineering, current and former undergraduate and graduate students, Director Digital Media, John Tubbs, and Prof. Hasegawa-Johnson, who have contributed to the development, support and direction of the ClassTranscribe project. We thank the UIUC IRB review board, and the CITL Data Analytics Group for their input and support.

The research reported here was supported by an Microsoft Corporation gift to the University of Illinois as part of the 2019 and 2020 Lighthouse Accessibility Microsoft-Illinois partnership, an award from Center for Innovative Teaching and Learning, and the Institute of Education Sciences, U.S. Department of Education through Grant R305A180211 to the Board of Trustees of the University of Illinois. The opinions expressed are those of the authors and do not represent views of the Institute or the U.S. Department of Education.

\section{References}

[1] Chirantan Mahipal and Lawrence Angrave and Yuren Xie and Biswadeep Chatterjee and Hongyu Wang and Zhengru Qian, “'What did I just miss?!' Presenting ClassTranscribe, an Automated Live-captioning and Text-searchable Lecture Video System, and Related Pedagogical Best Practices," in 2019 ASEE Annual Conference \& Exposition, Tampa, Florida, 2019.

[2] N. Bosch, E. Huang, L. Angrave, and M. Perry, "Modeling Improvement for Underrepresented Minorities in Online STEM Education," in Proceedings of the 27th ACM Conference on User Modeling, Adaptation and Personalization, 2019, pp. 327-335.

[3] L. Angrave, Z. Zhang, G. Henricks, and C. Mahipal, "Who Benefits? Positive Learner Outcomes from Behavioral Analytics of Online Lecture Video Viewing Using ClassTranscribe," in Proceedings of the 51st ACM Technical Symposium on Computer Science Education, Portland, OR, USA, 2020, pp. 1193-1199.

[4] "Women, Minorities, and Persons with Disabilities in Science and Engineering: 2019| NSF National Science Foundation.” [Online]. Available: https://ncses.nsf.gov/pubs/nsf19304/data. 
[Accessed: 02-Feb-2020]

[5] "Digest of Education Statistics, 2018" [Online]. Available:

https://nces.ed.gov/programs/digest/d18/tables/dt18_311.10.asp. [Accessed: 02-Feb-2020]

[6] J. M. Love, "Wording Matters: the Impact of Disability Identification in Post-Secondary Education," University of Wisconsin Milwaukee, 2017 [Online]. Available: https://dc.uwm.edu/etd/1662/.

[Accessed: 02-Feb-2020]

[7] B. Blaser, C. Bennett, R. E. Ladner, S. E. Burgstahler, and J. Mankoff, "Perspectives of Women with Disabilities in Computing," Cracking the Digital Ceiling: Women in Computing around the World, p. 159, 2019.

[8] W. Abeer and B. Miri, "Students' preferences and views about learning in a MOOC," Procedia-Social and Behavioral Sciences, vol. 152, no. 152, pp. 318-323, 2014.

[9] J. Reich and J. A. Ruipérez-Valiente, "The MOOC pivot," Science, vol. 363, no. 6423, pp. 130-131, Jan. 2019.

[10] D. Gamage, S. Fernando, and I. Perera, "Quality of MOOCs: A review of literature on effectiveness and quality aspects," in 2015 8th International Conference on Ubi-Media Computing (UMEDIA), 2015, pp. 224-229.

[11] T. E. Hall, A. Meyer, and D. H. Rose, Universal Design for Learning in the Classroom: Practical Applications. Guilford Press, 2012.

[12] R. D. Black, L. A. Weinberg, and M. G. Brodwin, "Universal Design for Learning and Instruction: Perspectives of Students with Disabilities in Higher Education," Exceptionality Education International, vol. 25, no. 2, pp. 1-16, 2015.

[13] J. Griful-Freixenet, K. Struyven, M. Verstichele, and C. Andries, "Higher education students with disabilities speaking out: perceived barriers and opportunities of the Universal Design for Learning framework," Disabil. Soc., vol. 32, no. 10, pp. 1627-1649, Nov. 2017.

[14] P. R. Clance and S. A. Imes, "The imposter phenomenon in high achieving women: Dynamics and therapeutic intervention," Group Dyn., 1978 [Online]. Available:

https://psycnet.apa.org/journals/pst/15/3/241/

[15] J. Langford and P. R. Clance, "The imposter phenomenon: recent research findings regarding dynamics, personality and family patterns and their implications for treatment," Psychotherapy: theory, research, practice, training, vol. 30, no. 3, p. 495, 1993.

[16] K. Cokley, S. McClain, A. Enciso, and M. Martinez, "An examination of the impact of minority status stress and impostor feelings on the mental health of diverse ethnic minority college students," J. Multicult. Couns. Devel., vol. 41, no. 2, pp. 82-95, 2013.

[17] C. C. Austin, E. M. Clark, M. J. Ross, and M. J. Taylor, "Impostorism as a mediator between survivor guilt and depression in a sample of African American college students," Coll. Stud. J., vol. 43, no. 4, pp. 1094-1109, 2009.

[18] A. Parkman, "The Imposter Phenomenon in Higher Education: Incidence and Impact," 1, vol. 16, no. 1, 2016, doi: 10.33423/jhetp.v16i1.1936. [Online]. Available: https://articlegateway.com/index.php/JHETP/article/view/1936. [Accessed: 03-Feb-2020]

[19] R. Felder, "Impostors everywhere," Chemical Engineering Education, vol. 22, no. 4, pp. 168-169, 1988.

[20] J. Weng and C. Murphy, "Bridging the Diversity Gap in Computer Science with a Course on Open Source Software," in 2018 Research on Equity and Sustained Participation in Engineering, Computing, and Technology (RESPECT), 2018, pp. 1-4.

[21] G. W. Corder and D. I. Foreman, Nonparametric Statistics: A Step-by-Step Approach. John Wiley \& Sons, 2014.

[22] D. H. Rose and A. Meyer, Teaching Every Student in the Digital Age: Universal Design for Learning. Association for Supervision and Curriculum Development, 1703 N. Beauregard St., 
Alexandria, VA 22311-1714 (Product no. 101042: \$22.95 ASCD members; \$26.95 nonmembers). Tel: 800-933-2723 (Toll Free); Tel: 703-578-9600; Fax: 703-575-5400; Web site:

http://www.ascd.org., 2002.

[23] M. V. Izzo and W. M. Bauer, "Universal design for learning: enhancing achievement and employment of STEM students with disabilities," Universal Access in the Information Society, vol. 14, no. 1, pp. 17-27, Mar. 2015. 